\title{
GLI inhibitor GANT61 kills melanoma cells and acts in synergy with obatoclax
}

\author{
KATEŘINA VLČKOVÁ ${ }^{1}$, JIRI RÉDA ${ }^{1}$, LUBICA ONDRUŠOVÁ ${ }^{1}$, \\ MOHAMMAD KRAYEM ${ }^{2}$, GHANEM GHANEM ${ }^{2}$ and JIRI VACHTENHEIM ${ }^{1}$ \\ ${ }^{1}$ Laboratory of Transcription and Cell Signaling, Institute of Medical Biochemistry and \\ Laboratory Diagnostics, First Faculty of Medicine, Charles University in Prague, Prague, Czech Republic; \\ ${ }^{2}$ LOCE-Institut Jules Bordet, Université Libre de Bruxelles, B-1000 Brussels, Belgium
}

Received April 11, 2016; Accepted May 26, 2016

DOI: 10.3892/ijo.2016.3596

\begin{abstract}
MEK kinase inhibitors (trametinib and selumetinib) or kinase inhibitors directed against mutated BRAF(V600E) (vemurafenib and dabrafenib) have initial encouraging effects in the treatment of melanoma but acquired resistance appears almost invariably after some months. Studies revealed mutually exclusive NRAS and BRAF activating mutations driving the MAPK/ERK pathway among human melanomas. Although combination therapy exerts significantly better antitumor cell efficacy, complete remission is rarely achieved. To employ an alternative approach, we have targeted the Hedgehog/GLI pathway, which is deregulated in melanomas, through the GLI1/2 inhibitor GANT61, alone or accompanied with the treatment by the BCL2 family inhibitor obatoclax in 9 melanoma cell lines. Thus, we targeted melanoma cells irrespective of their NRAS or BRAF mutational status. After GANT61 treatment, the cell viability was drastically diminished via apoptosis, as substantial nuclear DNA fragmentation was detected. In all tested melanoma cell lines, the combined treatment was more efficient than the application of each drug alone at the end of the cell growth with inhibitors. GANT61 was efficient also alone in most cell lines without the addition of obatoclax, which had only a limited effect when used as a single drug. In most cell lines, tumor cells were eradicated after 5-9 days of combined treatment in colony outgrowth assay. To conclude, GANT61 treatment might become a hopeful and effective anti-melanoma targeted therapy, especially when combined with the BCL2 family inhibitor obatoclax.
\end{abstract}

Correspondence to: Dr Jiri Vachtenheim, Laboratory of Transcription and Cell Signaling, Institute of Medical Biochemistry and Laboratory Diagnostics, First Faculty of Medicine, Charles University in Prague, Katerinska 32, Prague 2, 12108, Czech Republic

E-mail: jiri.vachtenheim@lf1.cuni.cz

Key words: GANT61, melanoma, Hedgehog signaling, GLI2, obatoclax

\section{Introduction}

Upregulation of the Hedgehog (HH/GLI) signaling pathway is responsible for the formation and progression of a number of human cancers through the aberrant activation of transcription factors GLI (1-3). Autocrine and paracrine ligand sonic Hedgehog (Shh) binds to Patched (PTCH) receptor, thereby relieving Patched repressive activity on the 7-transmembrane protein Smoothened (SMO), which in turn causes activation of the downstream effectors, zinc finger containing GLI transcription factors (4-7). Aberrant HH/GLI pathway activity has been initially identified in basal cell carcinomas and medulloblastomas (8) and later found to be deregulated in many common human cancers such as lung tumors, pancreas, colorectal, ovarian and prostate carcinomas, glioblastomas as well as melanomas $(5,7,9)$. Inhibitors of SMO vismodegib and cyclopamine have been used in many clinical trials. However, as the HH pathway can be upregulated non-canonically by direct activation of GLI factors by several signaling pathways (10-12), using the inhibitor of GLI activity GANT61 can overcome the possible ineffectiveness of upstream SMO inhibitors. GANT61 prevents the binding of GLIs to DNA (13) while fully preserving their expression.

GLI2 has been shown to control the invasiveness and metastatic potential and to contribute to the epithelial-tomesenchymal transition in melanoma (14). Melanomas express MITF (microphthalmia-associated transcription factor), a crucial factor in the pigment cell transcriptional circuitry, activating a large number of genes with various functions (15-17). GLI2 expression was reported to be inversely correlated with MITF expression. Thus, high GLI2 and low MITF levels characterize the invasive cell phenotype in melanoma $(18,19)$. High GLI2 expression in melanoma was achieved through the $\mathrm{HH}$ and TGF- $\beta$ /SMAD pathways $(20,21)$. HH/GLI1 signaling has an essential role in controlling self-renewal and tumor initiation of melanoma and GANT61 has been reported to reduce the number of melanomaspheres formed from cells with features of tumor initiating stem cells (22). However, despite the importance of HH/GLI pathway and GLI2 in the melanoma development, the data that would determine the effect of GANT61 on melanoma cell cultures in vitro is lacking. The treatment of melanoma by inhibitors against BRAF(V600E) 
or MAPK pathway were initially promising, but resistence appeared almost invariably after months through multiple mechanisms $(23,24)$. Other treatment approaches are therefore needed in melanoma.

In the present study, we undertook treatment of 9 melanoma cell lines with GANT61, a downstream Hedgehog/GLI pathway inhibitor, and performed a combined incubation of GANT61 with obatoclax, a BCL2 family inhibitor. We identify that melanoma cells are efficiently eliminated by GANT61 through apoptosis. GANT61 with obatoclax reveal synthetic lethality in cell lines in vitro as assessed by colony formation assays. Although the results require the in vivo verification, this targeted combined therapy is promising to be beneficial for melanoma patients irrespective of the BRAF or NRAS mutational status.

\section{Materials and methods}

Promoters and reporter assays. The $\Delta$ NGLI2 expression vector was previously described (25). The MITF promoterreporter construct has been described (26). Professor Fritz Aberger (University of Salzburg) provided the Patched promoter plasmid and Professor Rune Toftgard (Karolinska Institutet) the 12xGLI reporter plasmid. For reporter assays, melanoma cells were seeded in 12-well plates and transfected at 70-80\% confluency in fresh medium with LipoJet (SignaGen Laboratories, Rockville, MD, USA) according to the instructions of the manufacturer. Dual-luciferase assay kit (Promega, Madison, WI, USA) was used to determine promoter-reporter activity.

Cell lines. Melanoma cell lines MeWo, SK-MEL-3, SK-MEL-5, SK-MEL-28, WM35, WM1552C and SW13 line (human cervical carcinoma) were purchased from the American Type Culture Collection (ATCC; Manassas, VA, USA). Beu and $\mathrm{Hbl}$ cells were previously described (25). 501mel cell line was obtained from Dr Ruth Halaban (Yale University) and maintained in RPMI-1640 medium. Hbl and SW13 were grown in Dulbecco's modified Eagle's medium (DMEM) and all other cell lines were cultivated in EMEM medium. All types of media (Sigma-Aldrich, St. Louis, MI, USA) were supplemented with $10 \%$ fetal calf serum (FCS; Life Technologies, Carlsbad, CA, USA), L-glutamine and antibiotics (Sigma-Aldrich).

Cell viability assay. Cells (50,000/well) were seeded in 12-well plates (Nunc, Roskilde, Denmark) and treated the next day with $25 \mu \mathrm{M}$ GANT61 (Selleckchem, Munich, Germany) or DMSO (Sigma-Aldrich) (control) for $72 \mathrm{~h}$. Cultivation medium was removed and cell viability was determined with MTT cell viability assay kit (Sigma-Aldrich). Experiments were performed in duplicate and data are expressed as a mean of duplicate measurements (mean $\pm \mathrm{SD}$ ) in percentage of controltreated cells $(100 \%)$. Two independent experiments were carried out with similar results and one experiment is shown.

Cell proliferation assay. To perform colony outgrowth assay, subconfluent cells were trypsinised and seeded in 12-well plates (day 0). The next day, cell lines were treated with inhibitors at concentrations of $20 \mu \mathrm{M}$ GANT61 or $100 \mathrm{nM}$ obatoclax (Selleckchem), or their combination for the indicated time intervals (9 days maximum). The plates were fixed in 3\% paraformaldehyde solution in PBS and stained with $1 \%$ crystal violet. Two experiments were performed in duplicate. Results of both experiments were similar. The density of the remaining stained cells was quantified with the ImageJ software and the results of one experiment are shown.

Soft agar assay. Assay was performed in 60-mm dishes containing $0.6 \%$ lower layer of Noble agar (Difco, Radnor, PA, USA). Cells $\left(5 \times 10^{4}\right)$ were seeded in the upper agar $(0.4 \%$ agar in cultivation medium containing $15 \%$ FCS), overlayed with the cultivation medium with $15 \%$ FCS and DMSO or inhibitors. The media with inhibitors or control vehicle were refreshed twice a week. After 21 days colonies were stained with p-indonitrotetrazolium violet (Sigma-Aldrich), counted, photo-documented and quantified by the ImageJ software.

Detection of apoptotic cells. DNA fragmentation as a hallmark of advanced apoptosis was detected by flow cytometric and microscopic analyses. SK-MEL-3 and SK-MEL-5 cells were treated with $20 \mu \mathrm{M}$ GANT61 or DMSO (control) for $48 \mathrm{~h}$ and then trypsinized, combined with detached cells, washed with PBS, incubated in the DNA extraction buffer $(192 \mathrm{ml}$ of $0.2 \mathrm{M}$ $\mathrm{Na}_{2} \mathrm{HPO}_{4}$ with $8 \mathrm{ml}$ of $0.1 \mathrm{M}$ citric acid, $\mathrm{pH} 7.8$ ) for $10 \mathrm{~min}$ at room temperature, washed again with cold PBS, resuspended in PBS containing 5\% BSA (Merck, Darmstadt, Germany) and stained with propidium iodide (Sigma-Aldrich) $(20 \mu \mathrm{l}$ of $0.5 \mathrm{mg} / \mathrm{ml}$ solution per $1 \mathrm{ml}$ of cell suspension). RNAse (SigmaAldrich) was added simultaneously ( $2 \mu \mathrm{l}$ of the $20 \mathrm{mg} / \mathrm{ml}$ stock per $1 \mathrm{ml}$ of cell suspension). Cell histograms were acquired on a FACSCanto flow cytometer (Becton-Dickinson, Franklin Lakes, NJ, USA) with the FACSDiva VI acquisition and analysis software. To obtain microscopic images of apoptotic nuclei, cells were grown in 2-well chambers (NUNC) and treated with GANT61 as above. The remaining attached cells were then fixed with $3 \%$ paraformaldehyde in PBS. Slides were coverslipped with Vectashield mounting medium with DAPI (Vector Laboratories, Burlingame, CA, USA) to visualize the nuclei. Images were acquired on an Olympus BX61 microscope (Olympus, Tokyo, Japan).

Western blot analysis. Cell extracts for immunoblotting analysis were prepared by lysis the cells in RIPA buffer (1\% NP-40, $150 \mathrm{mM} \mathrm{NaCl}, 5 \mathrm{mM}$ EDTA, $0.5 \%$ sodium deoxycholate, $50 \mathrm{mM}$ Tris- $\mathrm{HCl} \mathrm{pH}$ 7.5, 0.1\% SDS) with added protease and phosphatase inhibitors: $1 \mu \mathrm{g} / \mathrm{ml}$ of leupeptin, aprotinin and pepstatin, and cOmplete and PhosStop (Roche Diagnostics, Mannheim, Germany) as recommended by the manufacturer. Protein extracts $(30 \mu \mathrm{g})$ in sample loading buffer (50 mM Tris-HCl pH 6.8; $2 \%$ sodium dodecyl sulphate; $100 \mathrm{mM}$ dithiotreitol; $10 \%$ glycerol; $0.1 \%$ bromophenol blue) were heated to $98^{\circ} \mathrm{C}$ for $2 \mathrm{~min}$ and proteins were separated on $10 \%$ polyacrylamide gels. After transfer onto the PVDF membrane (Millipore, Billerica, MA, USA), the membranes were blocked in 5\% Blotto (Santa Cruz Biotechnology, Dallas, TX, USA) in PBS containing 0.1\% Tween-20 (Sigma-Aldrich) at room temperature for $1 \mathrm{~h}$. The incubation with the primary antibodies against GLI2 (GTX46056), purchased from GeneTex (Irvine, CA, USA), MITF (Lab Vision, Fremont, CA, USA), or $\beta$-actin (AC-74) from Sigma-Aldrich was conducted 


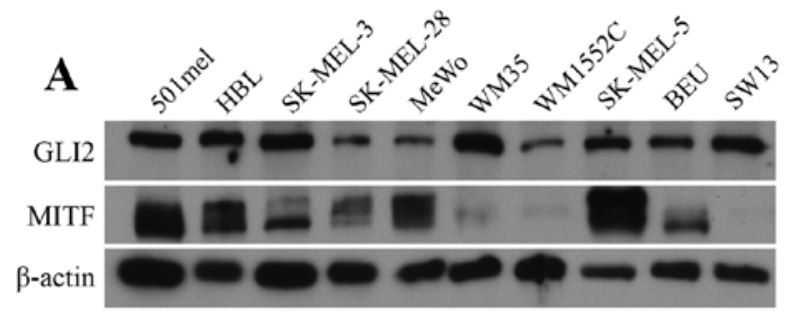<smiles>CN(C)c1ccccc1CN1CCCN(Cc2ccccc2N(C)C)C1</smiles>
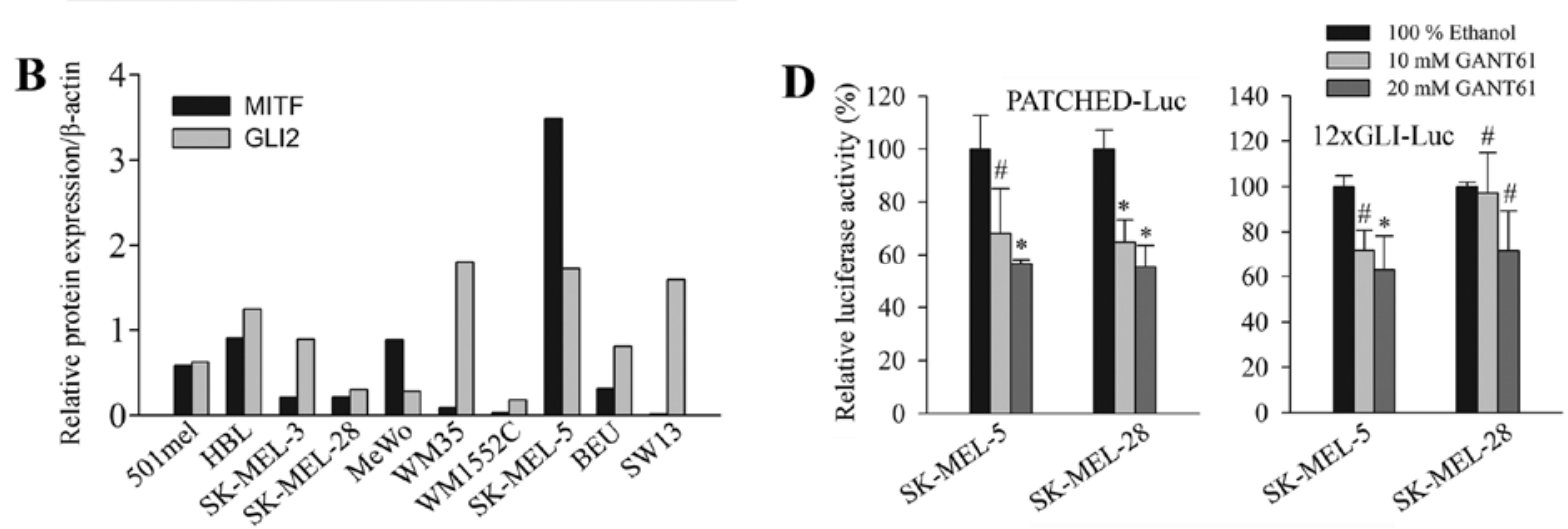

Figure 1. Expression of GLI2 and MITF in melanoma cell lines and inhibition of HH/GLI responsive promoters by GANT61. (A) Nine melanoma cell lines and a non-melanoma SW13 line were tested by western blotting with anti-GLI2 or anti-MITF antibody and control anti- $\beta$-actin antibody. (B) Quantification of western blot results from (A) expressed as relative expression of GLI2 or MITF corrected to the $\beta$-actin expression. Control cell line SW13 does not express MITF. (C) Chemical structure of GANT61. (D) Inhibition (\%) of HH/GLI-activated promoters by GANT61. Cells were transiently transfected with the promoter-luciferase plasmids Patched or $12 x \mathrm{GLI}(0.3 \mu \mathrm{g})$ together with the expression plasmid $\Delta \mathrm{NGLI} 2(0.7 \mu \mathrm{g})$ to increase the promoter activity. Cells were treated with GANT61 for $20 \mathrm{~h}$ before harvesting. Control value was set as $100 \%$ (luciferase arbitrary units). ${ }^{\#}$ Indicates statistically not significant; *P<0.05, statistically significant.

for several hours at room temperature, diluted $1: 1,000$ in the blocking solution. After washing in PBS-0.1\% Tween, $1 \mathrm{~h}$ incubation in the HRP-labelled secondary antibody (Cell Signaling Technology, Danvers, MA, USA) and washing, signals were detected by the Pierce ECL chemiluminiscent detection reagent (Thermo Fisher Scientific, Waltham, MA, USA). In western blot experiments determining the effect of GANT61 on MITF protein levels, GANT61 was left on cells for $20 \mathrm{~h}$ and the cells were harvested (Fig. 2).

Statistical analysis. Statistical comparison was carried out using the unpaired Student's t-test. Significance was set at $\mathrm{P}<0.05$. Standard error $(\mathrm{SE})$ is shown for triplicate or duplicate samples in promoter-reporter or cell viability assays, respectively. The combination index (CI) was calculated using CalcuSyn software. GLI2 and MITF proteins levels were corrected after densitometry (using AIDA image analyzer software) to western blots with the $\beta$-actin (control) expression (Figs. 1B and 2B).

\section{Results}

Expression of GLI2 in melanoma cell lines. GLI2 was shown to be an essential protein for maintaining the pro-oncogenic phenotype in melanoma (14). GLI2 expression was determined in melanoma cell lines and control non-melanoma cells SW13 (adrenal gland carcinoma) by western blotting. The BRAF and NRAS mutational status of melanoma cell lines used is provided in Table I (27-32). GLI2 was present in all cells examined (Fig. 1A). Expression was noted also in the $501 \mathrm{mel}$ cells, which were previously reported to be GLI2 negative (18).
In our recent study, these cells were also clearly GLI2 positive for both protein and mRNA, as detected by real-time PCR (25) and RT-PCR (data not shown). While the use of a different antibody might explain the difference in the western blot result, we can not clarify the discrepancy in the result of RNA level in 501mel cells at present. The western blot band density was quantitated and corrected to $\beta$-actin levels (Fig. 1B). In 6 of 9 cell lines, similar trend of inverse correlation between GLI2 and MITF was observed, as that previously reported (18). We observed this trend in different cell lines (with the exception of 501mel) than those employed by others (18). Since we have detected GLI2, a protein implicated in melanoma invasion and metastasis, in all cell lines in our panel, they are predicted to be good models for exploring the effect of GANT61. The chemical structure of GANT61 is shown in Fig. 1C.

GANT61 inhibits GLI2-dependent promoter reporters. To test whether GANT61 inhibits GLI-dependent transcription, we examined the activity of known $\mathrm{HH} / \mathrm{GLI}$ responsive promoter reporters, the $12 x \mathrm{xLI}$ arteficial super-promoter and the Patched promoter. After stimulation of these promoters by cotransfecting $\triangle$ NGLI2, the most effective GLI construct which was also the most effective stimulant for the newly discovered GLI2 target survivin promoter (25), increasing concentrations of GANT61 were added for $20 \mathrm{~h}$. The activity of both promoters was evidently decreased in two melanoma cell lines tested (Fig. 1D), albeit most results at $10 \mu \mathrm{M}$ concentration and one result at $20 \mu \mathrm{M}$ of GANT61 (the 12xGLI promoter in SK-MEL-28 cells) were not statistically significant (due to high SE values). Considering this observation, together with the finding of the uniform presence of GLI2 levels in mela- 
Table I. Mutational status of BRAF and NRAS in melanoma cell lines.

\begin{tabular}{llll}
\hline Cell line & \multicolumn{1}{c}{ BRAF } & NRAS & \\
\hline 501 mel & V600E & wt & Packer et al $(27)$ \\
Hbl & wt & wt & Herraiz et al (28) \\
SK-MEL-3 & V600E & wt & Hao et al (29) \\
SK-Mel-28 & V600E homozyg. & wt & Smalley et al (30) \\
MeWo & wt & & Singh et al (31) \\
WM35 & V600E & wt & Domenzain-Reyna et al (32) \\
WM1552C & V600E & wt & Smalley et al (30) \\
SK-MEL-5 & V600E homozyg. & wt & https://www.wistar.org/lab/meenhard-herlyn-dvm-dsc/page/ \\
& & melanoma-cell-lines-0 & Singh et al (31) \\
Beu & Not known & Not known & \\
\hline
\end{tabular}

A

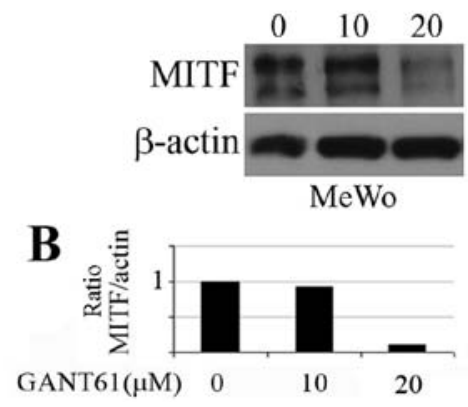

GANT61 $(\mu \mathrm{M})$
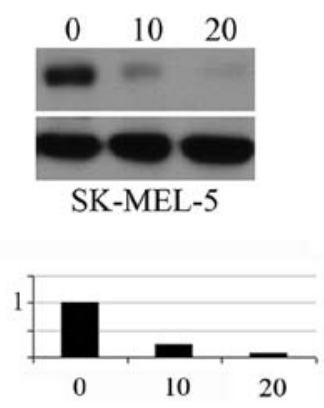

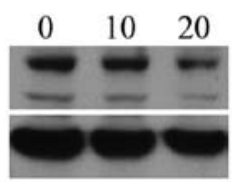

SK-MEL-28

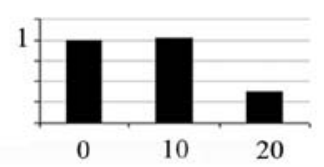

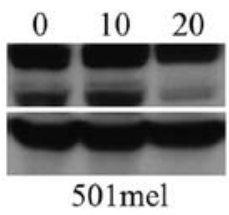

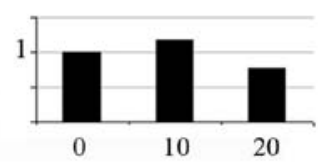

Figure 2. GANT61 treatment results in decrease of endogenous MITF protein level in melanoma cells. (A) Western blot analysis of cells incubated in medium with 0,10 and $20 \mu \mathrm{M}$ GANT61 for $20 \mathrm{~h}$, harvested, and protein lysates were analysed with the anti-MITF and control $\beta$-actin antibodies. (B) Quantification of western blots as relative expression of MITF compared to the $\beta$-actin expression.

noma cells (Fig. 1A), we hypothesized that GANT61 might possibly affect the growth of melanoma cells.

GANT61 decreases the MITF expression, cell viability and outgrowth of melanoma cells in soft agar. Since GLI2 expression was reported to be inversely correlated with the MITF expression (18) and GLI2 was described to repress MITF transcription (19), we expected that GANT61, a GLI1/2 inhibitor, would increase the MITF levels in melanoma cells. However, we observed no effect in MITF protein levels on western blots after treatment of cells with $10 \mu \mathrm{M}$ GANT61 (Fig. 2A) in 3 melanoma cell lines and a massive inhibition of MITF level in SK-MEL-5 cells even at $10 \mu \mathrm{M}$ (see Discussion) (Fig. 2A). The $20 \mu \mathrm{M}$ concentration inhibited MITF levels strongly in 3 cell lines and only negligibly in $501 \mathrm{mel}$ cells. These results are clearly evident after quantitation and correction of the MITF levels to $\beta$-actin levels (Fig. 2B).

We next addressed whether GANT61 has effects on cellular phenotype of melanoma cells. The viability of our panel of 9 melanoma cell lines and 3 non-melanoma cell lines was drastically diminished after 72-h incubation with $25 \mu \mathrm{M}$ GANT61. Only 5-35\% of viable cells remained. Only one of controls (A549 cells, lung carcinoma) was resistant as viability decreased by $<10 \%$ (Fig. 3A). The soft agar growth of the two most sensitive lines based on the viability assay, SK-MEL-3 and Beu, was examined. Whereas colony formation of SK-MEL-3 cells was completely abrogated by $20 \mu \mathrm{M}$ GANT61 after three weeks, Beu cells formed only small number of colonies (related to control) after quantitation of the dish images (Fig. 3D). We tested also $100 \mathrm{nM}$ obatoclax and its combination with GANT61 for clonogenic growth. Obatoclax completely abrogated soft agar colony outgrowth in SK-MEL-3 cells and it was more effective than GANT61 in Beu cells. Combined treatment completely prevented the colony formation in both cell lines (Fig. 3D), suggesting a synergistic effect in eradication of melanoma cells.

GANT61 induces massive apoptosis in melanoma cells. It is well established that GANT61 causes apoptosis in cancer cells (5,23-35). The possible accompanying caspase and PARP cleavage can be dependent on the cell type. To demonstrate whether GANT61 eliminates viability of melanoma cells through apoptosis, flow cytometric analysis was performed after 2 days of cultivation in at concentration of $20 \mu \mathrm{M}$. Massive sub-G1 phase indicating the DNA fragmentation was observed in SK-MEL-3 and SK-MEL-5 cells (Fig. 3B). Together with examining the remaining attached cells, we analysed also the detached cells, possibly 


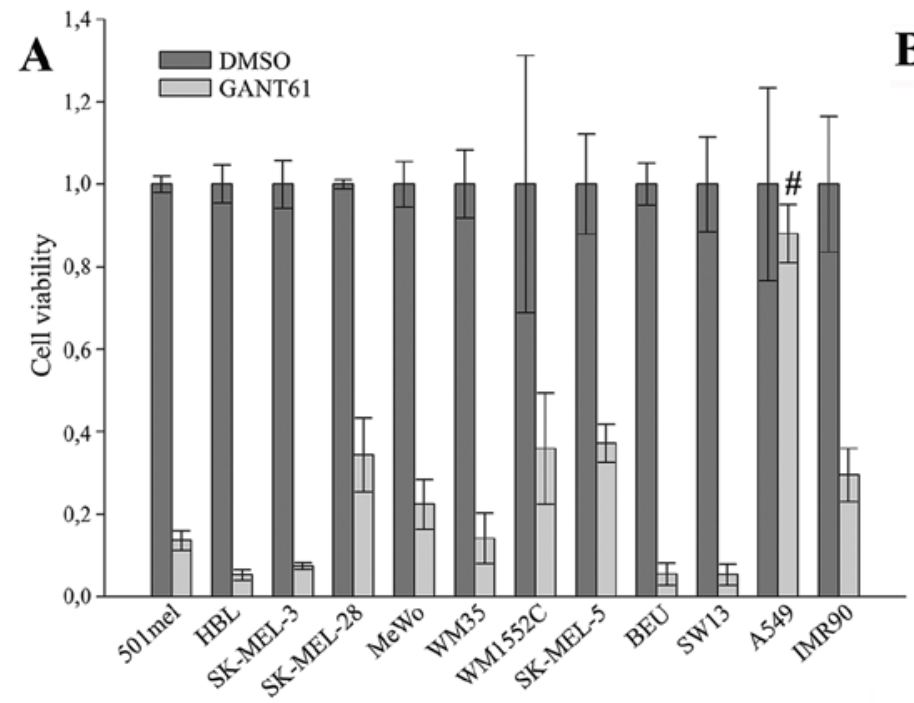

B
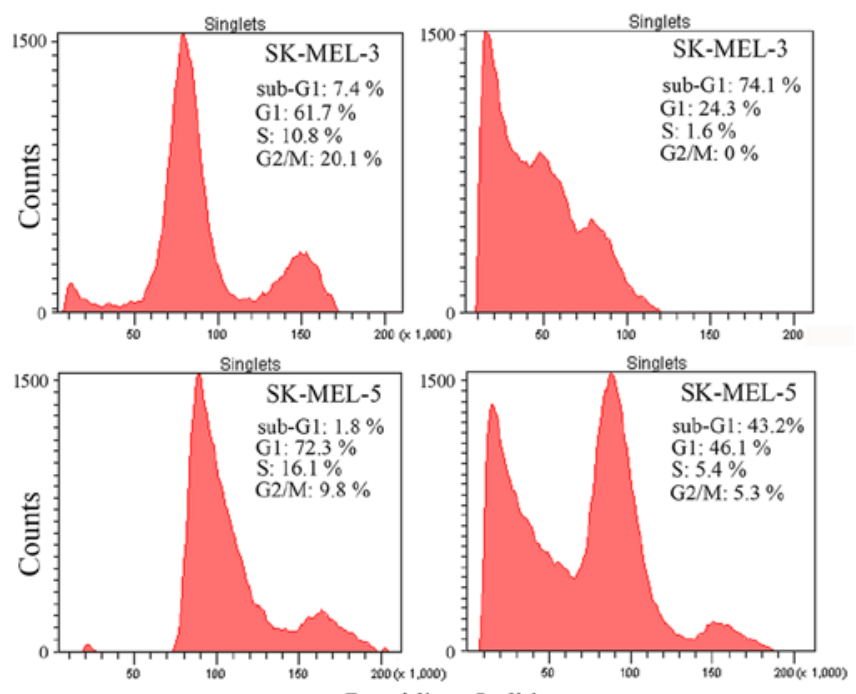

C

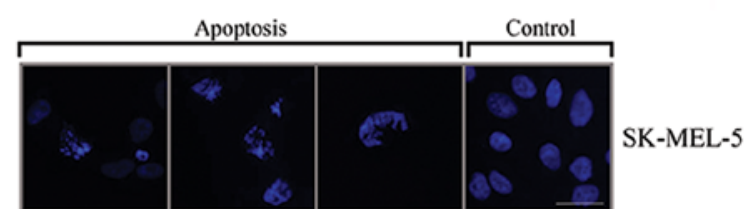

D
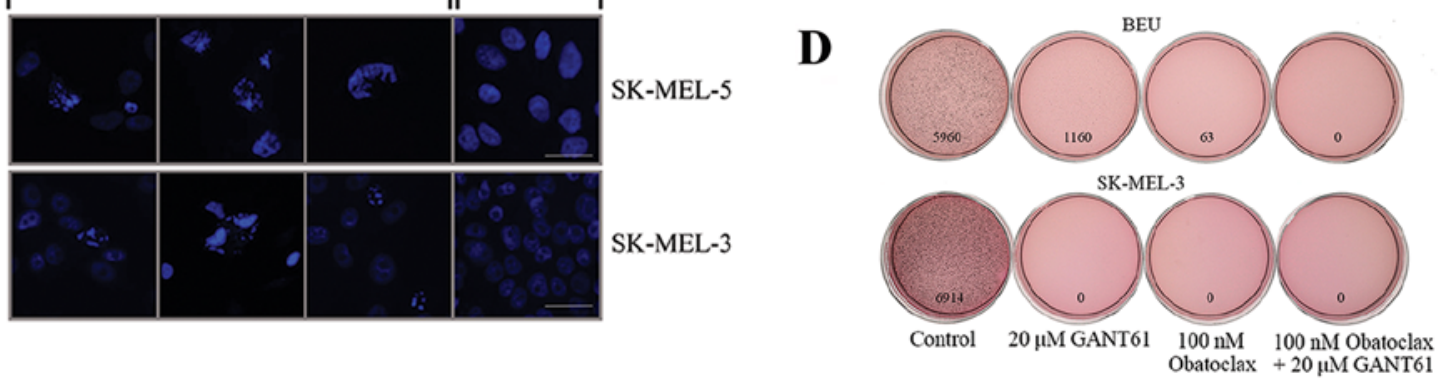

Figure 3. Decrease of viability, colony outgrowth in soft agar, and induction of apoptosis by GANT61. (A) Viability was tested on 9 melanoma cell lines and 3 control non-melanoma cell lines (SW13, A549 and IMR90) as indicated. After 3 days with GANT61 at $25 \mu$ M, cell viability was estimated by the MTT assay. (B) Two melanoma cell lines (SK-MEL-3 and SK-MEL-5) were treated with $20 \mu \mathrm{M}$ GANT61 for $48 \mathrm{~h}$. Apoptosis was measured by the propidium iodide method using flow cytometry. Both detached and remaining attached cells were measured. Cell cycle phases are indicated as inserts where sub-G1 phase represents the percentage of apoptotic cells. Left, control cells; right, cells treated with GANT61. (C) Apoptotic nuclei stained by DAPI. Three examples of apoptosis and one control are shown for each cell line. (D) Soft agar assay performed with two melanoma cell lines. The assay was carried out as described in Materials and methods and results of quantitation are depicted at the bottom of each dish.

explaining the appearance of such profound apoptosis. The high sensitivity of SK-MEL-3 correlated with the colony formation assays in which these cells appeared to be the most susceptible to GANT61 treatment (Fig. 4). To further substantiate that apoptosis was the mechanism of killing the melanoma cells, the same GANT61 treatment as used for flow cytometry was applied to cell monolayers and the remaining attached cells were mounted in DAPI-containing medium. Nuclei fragmentation and chromatin condensation indicating apoptosis were clearly seen, whereas normal nuclei were observed in control cells (Fig. 3C). Taken together these findings indicate that GANT61 induces powerful apoptosis in melanoma cells.

Obatoclax in synergy with GANT61 efficiently eradicate melanoma cells in vitro. Having shown that GANT61 can effectively abolish the survival of melanoma cells, we decided to investigate the possible synergistic effect of GANT61 when used with inhibitors related to melanoma treatment. Firstly, we studied whether selumetinib, a MEK inhibitor, and AZD5363, an AKT kinase inhibitor, could improve the efficacy of $20 \mu \mathrm{M}$ GANT61 in melanoma cells. We used $300 \mathrm{nM}$ concentration of both drugs, which is sufficiently high concentration considering that the $\mathrm{IC}_{50}$ values for both selumetinib and AZD5363 targets (MEK and ERK1/1 or AKT, respectively) are below $20 \mathrm{nM}$ (www. selleckchem.com). The experiments were designed similarly as shown in Fig. 4. However, the combined incubation of cell lines in the panel did not lead to improvement of the use of GANT61 alone (data not shown). Expectedly, selumetinib or AZD5363 alone (at $300 \mathrm{nM}$ concentration) did not reveal any considerable effect on any cell line even after 9 days of treatment (results not shown). We therefore used obatoclax, an inhibitor of the BCL2 family of anti-apoptotic proteins. Although it showed no effect on any cell line when tested alone (at $100 \mathrm{nM}$ concentration) (Fig. 4), obatoclax accelerated the effect of GANT61 treatment in 6 of 9 melanoma cell lines (501mel, Hbl, SK-MEL-28, MeWo, WM1552C and SK-MEL-5), whereas no additional effect was observed in the remaining 3 cell lines (SK-MEL-3, WM35 and Beu). In SK-MEL-3 (the most GANT61 sensitive melanoma cells), Beu melanoma cells and SW13 non-melanoma control cells, the cell death appeared even earlier when GANT61 was applied alone (Fig. 4). Combination index (CI) was calculated (Table II) revealing the 'antagonistic' effect in the Beu and SK-MEL-3 cell line, evidently due to the earlier effect 

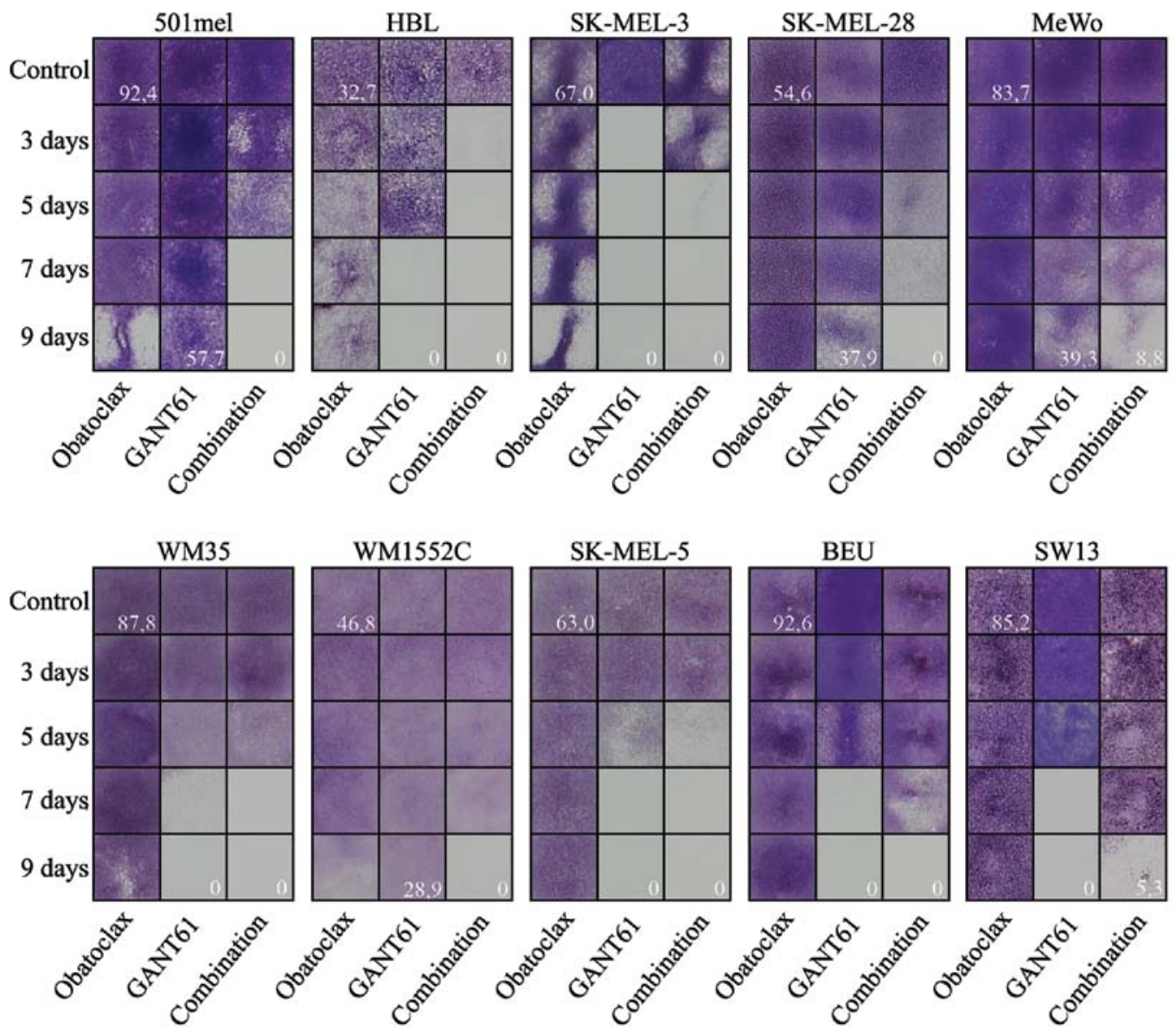

Figure 4. Proliferation of nine melanoma cell lines and SW13 cells in the presence of GANT61 (20 $\mu$ M), obatoclax (100 nM), or their combination. Colony formation assay was monitored for 9 days. Plates were then fixed, washed and stained with crystal violet. All wells were performed in duplicate and only one well is shown. The values between duplicates did not deviate by $>5 \%$. The values for day 1 (control) and day 9 (GANT61 and obatocalax) are indicated. Combination index has been calculated for each cell line and its values are indicated in Table II.

Table II. Combination index (CI) of the analyzed melanoma cell lines. ${ }^{a}$

\section{Cell line}

Combination index $(\mathrm{CI})$

\begin{tabular}{ll}
\hline 501mel & 0.091 \\
HBL & 0.14 \\
SK-MEL-3 & $1.798^{\mathrm{b}}$ \\
SK-MEL-28 & 0.121 \\
MeWo & 0.414 \\
WM35 & 0.95 \\
WM1552C & 0.812 \\
SK-MEL-5 & 0.216 \\
BEU & $1.88^{\mathrm{b}}$
\end{tabular}

SW13

(non-melanoma)

10.949

${ }^{\mathrm{a} C I}$ values between 0.9 and 1.1 indicate an additive effect; values between 0.7 and 0.9 are a moderate synergism; values $<0.7$ represent a strong synergism. Antagonism is represented by CI values $>1.1$. bPlease see the text for details.

of GANT61 alone than the combined treatment. Additive CI was calculated for WM35 cell line (Table II). Nonetheless, at the end of the experiment (day 9), all 9 melanoma cell lines revealed the best effect of GANT61 + obatoclax combination (the bottom right field in each block; Fig. 4).

Thus, GANT61 efficiently eliminated melanoma cells in the clonogenic growth assay and exhibited synthetically lethal effect with obatoclax in most melanoma cell lines.

\section{Discussion}

Malignant melanoma continues to increase in incidence worldwide. The treatment of advanced melanoma with low molecular weight inhibitors directed to mutated BRAF or MAPK inhibitors results in acquired resistance. It has been found that some kinase inhibitors induce a specific secretome increasing the tumor outgrowth and metastasis, supporting growth of cell clones with drug-resistance, actually promoting tumor progression after treatment (24). Although the combination of drugs improved the therapy, it remains questionable if MAPK signaling pathway, despite its deregulation virtually in all melanomas, is an ideal target for the therapy. More mechanisms are involved in the acquired resistance to the MAPK signaling in melanoma (23,36-39). Therefore, alternative approaches should also be considered.

GLI2 transcription factor has been recognized as important pro-invasive factor through its contribution to maintaining the 
cancer stem cell subpopulations and progression to metastasis in many cancers $(21,40-43)$. Many pro-oncogenic targets for GLI2 has been identified. Recently, we found that survivin is also a GLI2 target in about half of a large panel of human tumor cell lines, with several tumor lines being absolutely reliant on GLI2 for survivin epression (25). In keeping with this, both survivin expression and Hedgehog signaling are active during embryonic development and in tumor cells, while normal adult non-proliferating cells are silent or display very low activities of both HH/GLI and survivin expression. Thus, HH/GLI can widely contribute to the antiapoptotic activity through GLI2-directed survivin expression in cancer cells. GANT61 is a powerful and specific inhibitor of GLI1/2 factors activity. It also progresses into the clinic as a promising anticancer drug for many types of tumors. We therefore reasoned that it could constitute a possible effective agent in melanoma. We have shown here that it causes apoptosis in melanoma cells and inhibits their survival as assessed by colony formation assay. As the emerging data from both preclinical and clinical studies suggest that resistance is likely to occur following the monotherapy, we have successfully treated melanoma cells with GANT61 in combination with the BCL2 inhibitor obatoclax. Deregulated expression of BCL2 family proteins is known to play a central role in the resistance of melanoma to apoptosis (39).

In the present study, obatoclax accelerated the eradication of tumor cells in most melanoma lines (6 of 9), whereas we have not observed its toxicity against any melanoma cell line when used alone at $100 \mathrm{nM}$ concentration. In contrast, we found that either MEK inhibitor selumetinib or AKT inhibitor AZD-5363 did not synergize with GANT61 in eradication of melanoma cells. As obatoclax is effective towards the whole BLC2 family, the proposed enhancement of apoptosis induced by obatoclax may improve the results obtained with GANT61 and prevent the development of resistance in patients. To conclude, the presented combined treatment could be the basis for further research based on melanoma cell elimination through apoptosis and independently of BRAF or NRAS mutations and the activity of MAPK signaling.

Concomitantly, we estimated here the level of a pivotal transcription factor and melanoma oncogene MITF after GANT61 treatment. GLI2 has been demonstrated previously to repress transcription of MITF (19) and reciprocal expression of GLI2 and MITF is involved in the 'phenotype switching' model where high GLI2 functions to promote melanoma cell phenotypic plasticity and invasive behavior (18). Surprisingly, we found that the blockade of GLI2 activity by the addition of GANT61 does not increase the MITF protein, but rather has no effect or causes a decrease of its level. We therefore hypothesize that GLI2 may not be the MITF transcriptional repressor in every cell context, or GLI2 activity can eventually block the proteasomal degradation of the MITF protein by a yet unknown mechanism, similarly as observed for survivin in some tumor cell lines (25). However, although it is difficult to interpret these data at present, the findings together argue that in a specific cell context (SK-MEL-5 cells), GLIs are important to maintain the MITF cellular level. We speculate that the MITF level might not be a causative factor in the melanoma phenotype switching, but rather is a consequence that mirrors the phenotype changes governed by other factors. GLI2, on the other hand, activate genes that confer the invasive phenotype to melanoma cells and together with other factors such as SOX2, ZEB1, TWIST or SNAIL1 (44-46) could contribute to stem cell-like and metastasis prone properties of small subpopulations of cells in melanoma.

\section{Acknowledgements}

We thank Professor Fritz Aberger (University of Salzburg) for providing the Patched promoter plasmid and Professor Rune Toftgard (Karolinska Institutet) for the 12xGLI reporter plasmid. The present study was supported by IGA, Ministry of Health of the Czech Republic (grant NT/14005-3) and by the Institutional research project PRVOUK-P25/LF1/2 from the Charles University, Prague, Czech Republic.

\section{References}

1. Marini KD, Payne BJ, Watkins DN and Martelotto LG: Mechanisms of Hedgehog signalling in cancer. Growth Factors 29: 221-234, 2011.

2. Robbins DJ, Fei DL and Riobo NA: The Hedgehog signal transduction network. Sci Signal 5: re6, 2012.

3. Varjosalo M and Taipale J: Hedgehog: Functions and mechanisms. Genes Dev 22: 2454-2472, 2008

4. Katoh Y and Katoh M: Hedgehog target genes: Mechanisms of carcinogenesis induced by aberrant hedgehog signaling activation. Curr Mol Med 9: 873-886, 2009.

5. Gonnissen A, Isebaert S and Haustermans K: Targeting the Hedgehog signaling pathway in cancer: Beyond Smoothened. Oncotarget 6: 13899-13913, 2015.

6. McMillan R and Matsui W: Molecular pathways: The hedgehog signaling pathway in cancer. Clin Cancer Res 18: 4883-4888, 2012.

7. Li H, Li J and Feng L: Hedgehog signaling pathway as a therapeutic target for ovarian cancer. Cancer Epidemiol 40: 152-157, 2016.

8. Atwood SX, Chang AL and Oro AE: Hedgehog pathway inhibition and the race against tumor evolution. J Cell Biol 199: 193-197, 2012.

9. Stecca B, Mas C, Clement V, Zbinden M, Correa R, Piguet V, Beermann $F$ and Ruiz I Altaba A: Melanomas require HEDGEHOG-GLI signaling regulated by interactions between GLI1 and the RAS-MEK/AKT pathways. Proc Natl Acad Sci USA 104: 5895-5900, 2007.

10. Lauth M and Toftgård R: Non-canonical activation of GLI transcription factors: Implications for targeted anti-cancer therapy. Cell Cycle 6: 2458-2463, 2007.

11. Jenkins D: Hedgehog signalling: Emerging evidence for noncanonical pathways. Cell Signal 21: 1023-1034, 2009.

12. Shevde LA and Samant RS: Nonclassical hedgehog-GLI signaling and its clinical implications. Int J Cancer 135: 1-6, 2014.

13. Agyeman A, Jha BK, Mazumdar T and Houghton JA: Mode and specificity of binding of the small molecule GANT61 to GLI determines inhibition of GLI-DNA binding. Oncotarget 5: 4492-4503, 2014.

14. Alexaki VI, Javelaud D, Van Kempen LC, Mohammad KS, Dennler S, Luciani F, Hoek KS, Juàrez P, Goydos JS, Fournier PJ, et al: GLI2-mediated melanoma invasion and metastasis. J Natl Cancer Inst 102: 1148-1159, 2010.

15. Steingrímsson E, Copeland NG and Jenkins NA: Melanocytes and the microphthalmia transcription factor network. Annu Rev Genet 38: 365-411, 2004.

16. Hoek KS, Schlegel NC, Eichhoff OM, Widmer DS, Praetorius C, Einarsson SO, Valgeirsdottir S, Bergsteinsdottir K, Schepsky A, Dummer R, et al: Novel MITF targets identified using a two-step DNA microarray strategy. Pigment Cell Melanoma Res 21: 665-676, 2008.

17. Vachtenheim $\mathrm{J}$ and Borovanský J: 'Transcription physiology' of pigment formation in melanocytes: Central role of MITF. Exp Dermatol 19: 617-627, 2010. 
18. Javelaud D, Alexaki VI, Pierrat MJ, Hoek KS, Dennler S, Van Kempen L, Bertolotto C, Ballotti R, Saule S, Delmas V, et al: GLI2 and M-MITF transcription factors control exclusive gene expression programs and inversely regulate invasion in human melanoma cells. Pigment Cell Melanoma Res 24: 932-943, 2011.

19. Pierrat MJ, Marsaud V, Mauviel A and Javelaud D: Expression of microphthalmia-associated transcription factor (MITF), which is critical for melanoma progression, is inhibited by both transcription factor GLI2 and transforming growth factor- $\beta$. J Biol Chem 287: 17996-18004, 2012.

20. Dennler S, André J, Alexaki I, Li A, Magnaldo T, ten Dijke P, Wang XJ, Verrecchia F and Mauviel A: Induction of sonic hedgehog mediators by transforming growth factor-beta: Smad3-dependent activation of Gli2 and Gli1 expression in vitro and in vivo. Cancer Res 67: 6981-6986, 2007.

21. Javelaud D, Alexaki VI, Dennler S, Mohammad KS, Guise TA and Mauviel A: TGF- $\beta / \mathrm{SMAD} / \mathrm{GLI} 2$ signaling axis in cancer progression and metastasis. Cancer Res 71: 5606-5610, 2011.

22. Santini R, Vinci MC, Pandolfi S, Penachioni JY, Montagnani V, Olivito B, Gattai R, Pimpinelli N, Gerlini G, Borgognoni L, et al: Hedgehog-GLI signaling drives self-renewal and tumorigenicity of human melanoma-initiating cells. Stem Cells 30: 1808-1818, 2012.

23. Davies MA and Kopetz S: Overcoming resistance to MAPK pathway inhibitors. J Natl Cancer Inst 105: 9-10, 2013.

24. Obenauf AC, Zou Y, Ji AL, Vanharanta S, Shu W, Shi H, Kong X, Bosenberg MC, Wiesner T, Rosen N, et al: Therapy-induced tumour secretomes promote resistance and tumour progression. Nature 520: 368-372, 2015.

25. Vlčková $K$, Ondrušová L, Vachtenheim J, Réda J, Dundr P, Zadinová M, Žáková $\mathrm{P}$ and Poučková P: Survivin, a novel target of the Hedgehog/GLI signaling pathway in human tumor cells. Cell Death Dis 7: e2048, 2016.

26. Vachtenheim J, Sestáková B and Tuhácková Z: Inhibition of MITF transcriptional activity independent of targeting p300/ CBP coactivators. Pigment Cell Res 20: 41-51, 2007.

27. Packer LM, East P, Reis-Filho JS and Marais R: Identification of direct transcriptional targets of (V600E)BRAF/MEK signalling in melanoma. Pigment Cell Melanoma Res 22: 785-798, 2009.

28. Herraiz C, Journé F, Ghanem G, Jiménez-Cervantes C and García-Borrón JC: Functional status and relationships of melanocortin 1 receptor signaling to the cAMP and extracellular signal-regulated protein kinases 1 and 2 pathways in human melanoma cells. Int J Biochem Cell Biol 44: 2244-2252, 2012.

29. Hao H, Muniz-Medina VM, Mehta H, Thomas NE, Khazak V, Der CJ and Shields JM: Context-dependent roles of mutant B-Raf signaling in melanoma and colorectal carcinoma cell growth. Mol Cancer Ther 6: 2220-2229, 2007.

30. Smalley KS, Lioni M, Dalla Palma M, Xiao M, Desai B, Egyhazi S, Hansson J, Wu H, King AJ, Van Belle P, et al: Increased cyclin D1 expression can mediate BRAF inhibitor resistance in BRAF V600E-mutated melanomas. Mol Cancer Ther 7: 2876-2883, 2008.

31. Singh S, Davis R, Alamanda V, Pireddu R, Pernazza D, Sebti S, Lawrence $\mathrm{N}$ and Chellappan S: Rb-Raf-1 interaction disruptor RRD-251 induces apoptosis in metastatic melanoma cells and synergizes with dacarbazine. Mol Cancer Ther 9: 3330-3341, 2010.

32. Domenzain-Reyna C, Hernández D, Miquel-Serra L, Docampo MJ, Badenas C, Fabra A and Bassols A: Structure and regulation of the versican promoter: The versican promoter is regulated by AP-1 and TCF transcription factors in invasive human melanoma cells. J Biol Chem 284: 12306-12317, 2009.
33. Desch P, Asslaber D, Kern D, Schnidar H, Mangelberger D, Alinger B, Stoecher M, Hofbauer SW, Neureiter D, Tinhofer I, et al: Inhibition of GLI, but not Smoothened, induces apoptosis in chronic lymphocytic leukemia cells. Oncogene 29: 4885-4895, 2010.

34. Pan D, Li Y, Li Z, Wang Y, Wang P and Liang Y: Gli inhibitor GANT61 causes apoptosis in myeloid leukemia cells and acts in synergy with rapamycin. Leuk Res 36: 742-748, 2012.

35. Graab U, Hahn H and Fulda S: Identification of a novel synthetic lethality of combined inhibition of hedgehog and PI3K signaling in rhabdomyosarcoma. Oncotarget 6: 8722-8735, 2015.

36. Johannessen CM, Boehm JS, Kim SY, Thomas SR, Wardwell L, Johnson LA, Emery CM, Stransky N, Cogdill AP, Barretina J, et al: COT drives resistance to RAF inhibition through MAP kinase pathway reactivation. Nature 468: 968-972, 2010.

37. Nazarian R, Shi H, Wang Q, Kong X, Koya RC, Lee H, Chen Z, Lee MK, Attar N, Sazegar H, et al: Melanomas acquire resistance to B-RAF(V600E) inhibition by RTK or N-RAS upregulation. Nature 468: 973-977, 2010.

38. Poulikakos PI, Persaud Y, Janakiraman M, Kong X, Ng C, Moriceau G, Shi H, Atefi M, Titz B, Gabay MT, et al: RAF inhibitor resistance is mediated by dimerization of aberrantly spliced BRAF(V600E). Nature 480: 387-390, 2011.

39. Haq R, Yokoyama S, Hawryluk EB, Jönsson GB, Frederick DT, McHenry K, Porter D, Tran TN, Love KT, Langer R, et al: BCL2A1 is a lineage-specific antiapoptotic melanoma oncogene that confers resistance to BRAF inhibition. Proc Natl Acad Sci USA 110: 4321-4326, 2013

40. Zhang DW, Li HY, Lau WY, Cao LQ, Li Y, Jiang XF, Yang XW and Xue P: Gli2 silencing enhances TRAIL-induced apoptosis and reduces tumor growth in human hepatoma cells in vivo. Cancer Biol Ther 15: 1667-1676, 2014.

41. Kumar K, Raza SS, Knab LM, Chow CR, Kwok B, Bentrem DJ, Popovic R, Ebine K, Licht JD and Munshi HG: GLI2-dependent c-MYC upregulation mediates resistance of pancreatic cancer cells to the BET bromodomain inhibitor JQ1. Sci Rep 5: 9489, 2015.

42. Nagao-Kitamoto H, Nagata M, Nagano S, Kitamoto S, Ishidou Y, Yamamoto T, Nakamura S, Tsuru A, Abematsu M, Fujimoto Y, et al: GLI2 is a novel therapeutic target for metastasis of osteosarcoma. Int J Cancer 136: 1276-1284, 2015.

43. Soengas MS and Lowe SW: Apoptosis and melanoma chemoresistance. Oncogene 22: 3138-3151, 2003.

44. Caramel J, Papadogeorgakis E, Hill L, Browne GJ, Richard G, Wierinckx A, Saldanha G, Osborne J, Hutchinson P, Tse G, et al: A switch in the expression of embryonic EMT-inducers drives the development of malignant melanoma. Cancer Cell 24: 466-480, 2013.

45. Vandamme $\mathrm{N}$ and Berx G: Melanoma cells revive an embryonic transcriptional network to dictate phenotypic heterogeneity. Front Oncol 4: 352, 2014.

46. Denecker G, Vandamme N, Akay O, Koludrovic D, Taminau J, Lemeire K, Gheldof A, De Craene B, Van Gele M, Brochez L, et al: Identification of a ZEB2-MITF-ZEB1 transcriptional network that controls melanogenesis and melanoma progression. Cell Death Differ 21: 1250-1261, 2014. 